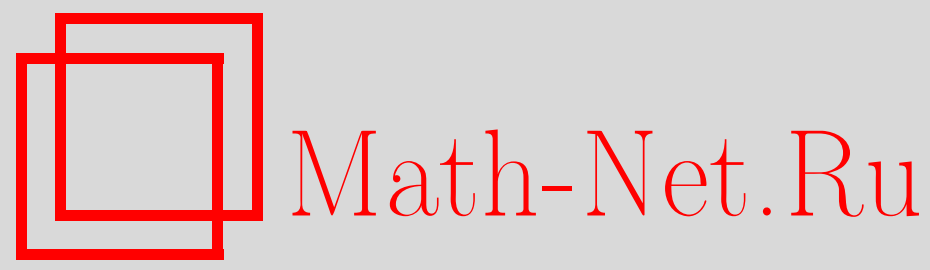

П. В. Ролдугин, О размерах подмножеств группы вычетов с необратимыми разностями элементов, Дискрет. матем., 2016, том 28, выпуск 3, 111-125

DOI: https://doi.org/10.4213/dm1386

Использование Общероссийского математического портала Math-Net.Ru подразумевает, что вы прочитали и согласны с пользовательским соглашением http://www.mathnet.ru/rus/agreement

Параметры загрузки:

IP : 54.196 .121 .252

26 апреля 2023 г., $15: 47: 53$

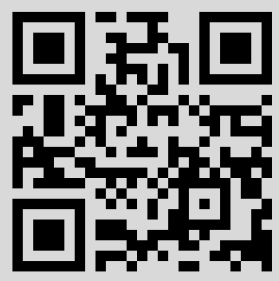




\title{
О размерах подмножеств группы вычетов с необратимыми разностями элементов
}

\author{
() 2016 г. П. В. Ролдугин*
}

В статье рассматриваются такие подмножества $I \subset\{0, \ldots, d-1\}$, что НОД $(n-m, d) \neq 1$ для любых $n, m \in I$. Эти подмножества названы множествами нетривиальных разностей. Пусть $d>1$ и $d_{1}-$ наименьший простой делитель $d$. Доказано, что наибольшее значение мощности множества нетривиальных разностей равно $d / d_{1}$. Множества нетривиальных разностей, в которых не все разности элементов кратны одному и тому же простому делителю $d$, названы неэлементарными. Пусть $t-$ количество простых делителей числа $d$. Показано, что при $t \leqslant 2$ неэлементарных множеств не существует. Доказано, что минимальное неэлементарное множество может иметь любой порядок в отрезке $\overline{3, t}$. Найдены нижняя и верхняя оценки наибольшей мощности неэлементарных множеств.

Ключевые слова: группы вычетов, разности элементов, необратимые элементы, мощности подмножеств

\section{1. Введение}

Используем обозначение $N_{d}=\{0, \ldots, d-1\}$. Подмножество $I \subset N_{d}$ назовем множеством нетривиальных разностей, если НОД $(n-m, d) \neq 1$ для любых различных $n, m \in I$. По определению пустое множество не будем считать множеством нетривиальных разностей.

Множества нетривиальных разностей являются обобщением так называемых несмежных множеств (nonadjacent sets, [3]), возникающих при построении алгоритма дискретного логарифмирования в случае малого двоичного веса экспоненты. Для несмежных множеств требуется, чтобы разность любых двух элементов не равнялась 1. Кроме того, множество нетривиальных разностей отражает связь между группой $\left(N_{d},+\right)$ и полугруппой $\left(N_{d}, \cdot\right)$, поскольку $M \subset N_{d}$ является множеством нетривиальных разностей тогда и только тогда, когда разность любых двух элементов из $M$ необратима в $\left(N_{d}, \cdot\right)$

Всюду далее будем считать, что $d>1$ и $d=d_{1}^{a_{1}} \ldots d_{t}^{a_{t}}, a_{i}>0, d_{1}, \ldots, d_{t}$ - простые, $d_{1}<d_{2}<\ldots<d_{t}, t \geqslant 1$. В этих обозначениях условие $\operatorname{HOД}(n-m, d) \neq 1$ эквивалентно существованию такого $i \in \overline{1, t}$, что $n \bmod d_{i}=m \bmod d_{i}$. Ниже

*Место работы: Московский государственный технический университет радиотехники, электроники и автоматики, e-mail: PavRoldugin@rambler.ru 
часто используется китайская теорема об остатках, согласно которой целые числа $m_{1}, \ldots, m_{t}, 0 \leqslant m_{i} \leqslant d_{i}^{a_{i}}-1, i \in \overline{1, t}$, однозначно определяют такое число $n \in N_{d}$, что $n \bmod d_{i}^{a_{i}}=m_{i}, i \in \overline{1, t}$. Отсюда вытекает еще одна интерпретация множества нетривиальных разностей для случая, когда $a_{1}=\ldots=a_{t}=1$. По китайской теореме об остатках множество $N_{d}$ взаимно однозначно соответствует множеству $B_{t}$ наборов $\left(x_{1}, \ldots, x_{t}\right)$, где $0 \leqslant x_{i} \leqslant d_{i}-1$. Гранью в $B_{t}$ назовем совокупность наборов, имеющих хотя бы одну одинаковую координату. Множество нетривиальных разностей отвечает такой совокупности наборов в $B_{t}$, в которой любые два элемента лежат в одной грани.

Пусть $\mu(d)$ - наибольшая мощность множества нетривиальных разностей из $N_{d}$. Все множество $N_{d}$ делится на $d_{1}$ непересекающихся подмножеств $N_{d}=I_{0} \cup I_{1} \cup$ $\ldots \cup I_{d_{1}-1}$, где $I_{j}=\left\{n \in N_{d}: n \bmod d_{1}=j\right\}, j \in \overline{0, d_{1}-1}$. Заметим, что каждое из $I_{j}$, очевидно, является множеством нетривиальных разностей. Отсюда $\mu(d) \geqslant \frac{d}{d_{1}}$. Несложно получить и обратное неравенство; более того, верно следующее утверждение.

Утверждение 1. Для любого $d>1$ справедливо равенство $\mu(d)=\frac{d}{d_{1}}$. Кроме того, любое множество нетривиалъных разностей в $N_{d}$ мощности $\frac{d}{d_{1}}$ совпадает с $I_{j} n p u$ некотором $j \in \overline{1, d_{1}-1}$.

Доказательство. Пусть $A=\left\{n^{(1)}, \ldots, n^{(\mu(d))}\right\}-$ множество нетривиальных разностей мощности $\mu(d)$. Без ограничения общности считаем, что $n^{(1)}>\ldots>n^{(\mu(d))}$. Поскольку НОД $\left(d, n^{(j)}-n^{(j+1)}\right) \neq 1$ для любого $j \in \overline{1, \mu(d)-1}, d_{1}-$ наименьший из простых делителей $d$ и $n^{(j)}-n^{(j+1)}>0$, то $n^{(j)}-n^{(j+1)} \geqslant d_{1}$. Кроме того, НОД $\left(d, n^{(\mu(d))}-n^{(1)}\right) \neq 1$, поэтому НОД $\left(d, d+n^{(\mu(d))}-n^{(1)}\right) \neq 1 ;$ учитывая, что $d+n^{(\mu(d))}-n^{(1)}>0$, получаем $d+n^{(\mu(d))}-n^{(1)} \geqslant d_{1}$. Далее имеем

$$
\begin{aligned}
d & =d+n^{(1)}+\ldots+n^{(\mu(d))}-n^{(1)}-\ldots-n^{(\mu(d))}= \\
& =\left(n^{(1)}-n^{(2)}\right)+\left(n^{(2)}-n^{(3)}\right)+\ldots+\left(n^{(\mu(d)-1)}-n^{(\mu(d))}\right) \\
& +\left(d+n^{(\mu(d))}-n^{(1)}\right) \geqslant \mu(d) \cdot d_{1}
\end{aligned}
$$

Значит, $\mu(d) \leqslant \frac{d}{d_{1}}$. Учитывая обратное неравенство, находим, что $\mu(d)=\frac{d}{d_{1}}$.

Но последнее означает, что в (1) неравенство обращается в равенство, что может быть только в случае, когда $n^{(j)}-n^{(j+1)}=d_{1}$ для любого $j \in \overline{1, \mu(d)-1}$ и $d+$ $n^{(\mu(d))}-n^{(1)}=d_{1}$. Так как $n^{(1)}<d$, то $d_{1}=d+n^{(\mu(d))}-n^{(1)}>n^{(\mu(d))}$. Следовательно, $n^{(j)}=n^{(\mu(d))}+(\mu(d)-j) \cdot d_{1}$ для любого $j \in \overline{1, \mu(d)}$, т.е. множество $A$ совпадает с множеством $I_{\mu(d)}$.

Как показывает утверждение 1 , самыми большими множествами нетривиальных разностей в $N_{d}$ являются множества, в которых разность любых двух элементов делится на $d_{1}$. Однако этот случай тривиален, и интерес представляют множества нетривиальных разностей более сложной структуры. Такие множества существуют, например $\{0,2,5\}$ в $N_{30}$.

Введем определение. Назовем подмножество $I \subset N_{d}$ элементарным по модулю $d_{i}$ (или просто элементарным), если существует такое $0 \leqslant \rho_{0} \leqslant d_{i}-1$, что каждый 
элемент представляется в виде $\rho_{0}+d_{i} \cdot \rho$, где $0 \leqslant \rho \leqslant \frac{d}{d_{i}}-1$. Иначе говоря, элементарное подмножество по модулю $d_{i}$ это любое непустое подмножество произвольного смежного класса по циклической подгруппе в $\left(N_{d},+\right)$, порожденной элементом $d_{i}$.

Далее в статье доказано, что неэлементарные множества нетривиальных разностей существуют только при $t \geqslant 3$; более того, минимальное неэлементарное множество нетривиальных разностей (т.е. неэлементарное множество, любое собственное подмножество которого является элементарным) мощности $k$ существует тогда и только тогда, когда $3 \leqslant k \leqslant t$. Кроме того, для неэлементарных множеств нетривиальных разностей получены верхняя и нижняя оценка наибольшего значения мощности.

\section{2. Неэлементарные множества нетривиальных разностей}

Обозначим через $R(d)$ совокупность всех неэлементарных множеств нетривиальных разностей. Теоретико-множественное отношение вложенности позволяет рассматривать семейство $R(d)$ как частично упорядоченное множество. Сначала рассмотрим вопрос о минимальных элементах этого множества, т. е. таких $I \in R(d)$, что не существует собственного подмножества $J \subset I$, удовлетворяющего условию $J \in R(d)$.

Сразу исключим случай $t=1$, поскольку тогда, очевидно, в $N_{d}$ существуют только элементарные множества нетривиальных разностей. Как показывает следующее утверждение, неэлементарные множества нетривиальных разностей существуют только в случае $t \geqslant 3$.

Утверждение 2. Если число $d$ таково, что $t=2$, то любое множество нетривиальных разностей в $N_{d}$ является элементарным.

Доказательство. Пусть $I \subset N_{d}$ - множество нетривиальных разностей. Проведем доказательство индукцией по $|I|$. Любое одноэлементное или двухэлементное множество $I$, очевидно, является элементарным. Пусть любое $q$-элементное множество нетривиальных разностей элементарно и $|I|=q+1, q \geqslant 2$. Возьмем в $I$ произвольный элемент $n$, тогда $I \backslash\{n\}$ элементарно. Без ограничения общности считаем, что $I \backslash\{n\}$ элементарно по модулю $d_{1}$. Рассмотрим два случая. В первом случае $d_{2} \mid n-m$ для любого элемента $m \in I \backslash\{n\}$, тогда возьмем произвольные $n^{(1)}, n^{(2)} \in I \backslash\{n\}$ (они существуют, так как $|I| \geqslant 3$ ) и, поскольку $d_{2} \mid n-n^{(1)}$ и $d_{2} \mid n-n^{(2)}$, получим $d_{2} \mid n^{(1)}-n^{(2)}$. Значит, $I$ является элементарным по модулю $d_{2}$. Во втором случае существует такое $m \in I \backslash\{n\}$, что $d_{1} \mid n-m$. Тогда $d_{1} \mid \tilde{n}-m$ для любого $\tilde{n} \in I \backslash\{n\}$ и $d_{1} \mid n-m$, поэтому $d_{1} \mid n-\tilde{n}$ и множество $I$ является элементарным по модулю $d_{1}$.

Следующее утверждение дает полное описание порядков минимальных элементов в $R(d)$.

Утверждение 3. Пусть число $d$ таково, что $t \geqslant 3$. Если $I \in R(d)$ минимально, то $3 \leqslant|I| \leqslant t$. Кроме того, для любого $w \in \overline{3, t}$ найдется такое минимальное $T_{w} \in R(d)$, чmo $\left|T_{w}\right|=w$. 
Доказательство. Пусть $I \in R(d)$ минимально. Тогда неравенство $|I| \geqslant 3$ очевидно. Докажем второе неравенство.

Выберем любой элемент $a \in I$. Если существует такое $d_{i}, i=\overline{1, t}$, что $a \bmod d_{i}=b \bmod d_{i}$ для всех $b \in I \backslash\{a\}$, то $I-$ элементарное множество, поскольку для произвольных $n, m \in I$ разность $n-m=(n-a)-(m-a)$ делится на $d_{i}$. Следовательно, для каждого $d_{i}, i=\overline{1, t}$, найдется такое $n^{(i)} \in I \backslash\{a\}$, что $a \bmod d_{i} \neq n^{(i)} \bmod d_{i}$. Рассмотрим множество $J=\left\{a, n^{(1)}, \ldots, n^{(t)}\right\}$. Тогда $|J| \leqslant t+1$ (здесь неравенство, поскольку не все из $n^{(1)}, \ldots, n^{(t)}$ обязаны быть различными). Это множество, очевидно, не является элементарным. Если $J \neq I$, то $I$ не минимально. Следовательно, $|I| \leqslant t+1$. Пусть $|I|=t+1$. Далее, пусть все подмножества мощности $t$ в $I$ являются элементарными. Но таких подмножеств $t+1$ штук, поэтому среди них найдутся два подмножества $I^{\prime}, I^{\prime \prime} \subset I$, элементарные по одному и тому же модулю $d_{k}$. Очевидно, что $I=I^{\prime} \cup I^{\prime \prime}$, поэтому все множество $I$ оказывается элементарным по модулю $d_{k}$, что противоречит условию $I \in R(d)$. Значит, в множестве $I$ найдется неэлементарное подмножество мощности $t$, что, в свою очередь, противоречит минимальности $I$. Следовательно, случай $|I|=t+1$ невозможен, поэтому $|I| \leqslant t$.

Докажем вторую часть утверждения. Пусть $w \in \overline{3, t}$. Поскольку $d_{1}<\ldots<d_{t}$ - простые числа, $w<d_{w}$ и поэтому $w<d_{w+1}<\ldots<d_{t}$. Рассмотрим $w$ чисел $n^{(1)}, \ldots, n^{(w)}$ из $N_{d}$, определяемых следующим образом. Число $n^{(j)}, j \in \overline{1, w}$, однозначно задается по китайской теореме своими остатками: $n^{(j)} \bmod d_{i}^{a_{i}}=0$ при $i \in \overline{1, j-1} \cup \overline{j+1, w} ; n^{(j)} \bmod d_{j}^{a_{j}}=1 ; n^{(j)} \bmod d_{i}^{a_{i}}=j$ при $i \in \overline{w+1, t}$. Последнее равенство корректно, поскольку $w<d_{i}$ при $i \in \overline{w+1, t}$. Для большей ясности остатки чисел $n^{(1)}, \ldots, n^{(w)}$ от деления на $d_{1}^{a_{1}}, \ldots, d_{t}^{a_{t}}$ сведены в таблицу 1.

\begin{tabular}{|c|c|c|c|c|c|c|c|}
\hline & $i=1$ & $i=2$ & $\ldots$ & $i=w$ & $i=w+1$ & $\ldots$ & $i=t$ \\
\hline$n^{(1)} \bmod d_{i}^{a_{i}}$ & 1 & 0 & $\ldots$ & 0 & 1 & $\ldots$ & 1 \\
\hline$n^{(2)} \bmod d_{i}^{a_{i}}$ & 0 & 1 & $\ldots$ & 0 & 2 & $\ldots$ & 2 \\
\hline$\ldots$ & \multicolumn{6}{|c|}{$\ddots$} & . \\
\hline$n^{(w)} \bmod d_{i}^{a_{i}}$ & 0 & 0 & $\ldots$ & 1 & $w$ & $\ldots$ & $w$ \\
\hline
\end{tabular}

Таблица 1.

Из таблицы видно, что $n^{(u)} \bmod d_{k}^{a_{k}}=0$ и $n^{(v)} \bmod d_{k}^{a_{k}}=0$ для любых $n^{(u)}$ и $n^{(v)}, u \neq v$, и любого $k \in \overline{1, w} \backslash\{u, v\}$, а значит, $d_{k}$ делит $n^{(u)}-n^{(v)}$. Хотя бы одно такое $k$ найдется, поскольку $w \geqslant 3$. Значит, множество $T_{w}=\left\{n^{(1)}, \ldots, n^{(w)}\right\}$ является множеством нетривиальных разностей. Далее, множество $T_{w}$ не является элементарным, поскольку $d_{1}$ не делит $n^{(1)}-n^{(2)}, d_{2}$ не делит $n^{(2)}-n^{(3)}$ и так далее, $d_{w}$ не делит $n^{(w)}-n^{(1)} ;$ любое $d_{i}, i \in \overline{w+1, t}$, не делит любую из разностей (здесь используется, что $w<d_{i}$ при $i \in \overline{w+1, t}$ ). Наконец, рассмотрим произвольное собственное подмножество $J$ множества $T_{w}$. Пусть $J$ не содержит $n^{(j)}, j \in \overline{1, w}$, тогда все элементы из $J$ и, соответственно, разности пар элементов делятся на $d_{j}$. Получаем, что $J$ - элементарное множество и, следовательно, $T_{w}$ - минимальное множество из $R(d)$ мощности $w$. 
Теперь рассмотрим вопрос о наибольшем значении мощности множества из $R(d)$. Обозначим эту величину через $w_{\max }(d)$.

Утверждение 4. Пусть число d таково, что $t \geqslant 3$. Тогда

$$
w_{\max }(d) \leqslant \frac{d}{d_{1}} \cdot\left(1-\prod_{i=2}^{t}\left(1-\frac{2}{d_{i}^{a_{i}}}\right)\right) .
$$

Доказательство. Поставим в соответствие каждому $n \in N_{d}$ число $\xi(n) \in N_{d}$, определяемое следующим образом: $\xi(n)-$ это такое число, что $\xi(n) \bmod d_{i}^{a_{i}}=$ $\left(n+d_{1}-1-\left(n \bmod d_{1}\right)\right) \bmod d_{i}^{a_{i}}, i \in \overline{1, t}$. Число $\xi(n)$ определяется однозначно согласно китайской теореме об остатках. Имеем

$$
\begin{aligned}
& \xi(n) \bmod d_{1}=\left(\xi(n) \bmod d_{1}^{a_{1}}\right) \bmod d_{1}= \\
& =\left(\left(n+d_{1}-1-\left(n \bmod d_{1}\right)\right) \bmod d_{1}^{a_{1}}\right) \bmod d_{1}=d_{1}-1 .
\end{aligned}
$$

Значит, $\xi(n) \in I_{d_{1}-1}$ и $\xi: N_{d} \rightarrow I_{d_{1}-1}$. Рассмотрим некоторые свойства этого отображения.

Лемма 1. При фиксированном $j=\overline{0, d_{1}-1}$ сужение отображения $\xi$ на множество $I_{j}$ является биекиией.

Доказательство. Зафиксируем $j=\overline{0, d_{1}-1}$. Если $n, \tilde{n} \in I_{j}$ и $n \neq \tilde{n}$, то $\xi(n) \neq$ $\xi(\tilde{n})$, поскольку в противном случае при каждом $i \in \overline{1, t}$

$$
\left(n+d_{1}-1-\left(n \bmod d_{1}\right)\right) \bmod d_{i}^{a_{i}}=\left(\tilde{n}+d_{1}-1-\left(\tilde{n} \bmod d_{1}\right)\right) \bmod d_{i}^{a_{i}} .
$$

Учитывая, что $n \bmod d_{1}=\tilde{n} \bmod d_{1}=j$, получаем $n \bmod d_{i}^{a_{i}}=\tilde{n} \bmod d_{i}^{a_{i}}$ для каждого $i \in \overline{1, t}$, и вновь по китайской теореме об остатках $n=\tilde{n}$. Значит, отображение $\xi$ из $I_{j}$ в $I_{d_{1}-1}$ инъективно, а поскольку $\left|I_{j}\right|=\left|I_{d_{1}-1}\right|$, то и биективно.

Пусть $A-$ множество нетривиальных разностей. Пусть, далее, $A_{j}=A \cap I_{j}$, $j \in \overline{0, d_{1}-1}$, и $\xi\left(A_{j}\right)$ - образ множества $A_{j}$ при отображении $\xi$. Согласно лемме $1,\left|\xi\left(A_{j}\right)\right|=\left|A_{j}\right|, j \in \overline{0, d_{1}-1}$. Отметим для понимания, что множество $\xi\left(A_{d_{1}-1}\right)$ попросту совпадает с $A_{d_{1}-1}$, поскольку на $I_{d_{1}-1}$ отображение $\xi$ является тождественным.

Лемма 2. Если $l, j \in \overline{0, d_{1}-1}, l \neq j, \operatorname{mo} \xi\left(A_{l}\right) \cap \xi\left(A_{j}\right)=\varnothing$.

Доказательство. Предположим, что при некоторых $l \neq j, l, j \in \overline{0, d_{1}-1}$, $\xi\left(A_{l}\right) \cap \xi\left(A_{j}\right) \neq \varnothing$. Тогда существуют такие $n \in A_{l}$ и $\tilde{n} \in A_{j}$, что $\xi(n)=\xi(\tilde{n})$. Поскольку, в частности, $n, \tilde{n} \in A$ и $A$ является множеством нетривиальных разностей, то существует такое $k \in \overline{1, t}$, что $n \bmod d_{k}=\tilde{n} \bmod d_{k}$. Рассмотрим равенство $\xi(n)=\xi(\tilde{n})$ по модулю $d_{k}^{a_{k}}$ :

$$
\left(n+d_{1}-1-\left(n \bmod d_{1}\right)\right) \bmod d_{k}^{a_{k}}=\left(\tilde{n}+d_{1}-1-\left(\tilde{n} \bmod d_{1}\right)\right) \bmod d_{k}^{a_{k}} .
$$

Учитывая, что $n \bmod d_{1}=l$ и $\tilde{n} \bmod d_{1}=j$, получаем равенство $(n-l) \bmod$ $d_{k}^{a_{k}}=(\tilde{n}-j) \bmod d_{k}^{a_{k}}$. Но тогда $(n-l) \bmod d_{k}=(\tilde{n}-j) \bmod d_{k}$ и, поскольку $n \bmod d_{k}=\tilde{n} \bmod d_{k}$, получаем $l \bmod d_{k}=j \bmod d_{k}$. Последнее невозможно, так как $l, j<d_{1} \leqslant d_{k}$ и $i \neq j$. 
Из леммы 2 следует, что $\xi\left(A_{0}\right), \ldots, \xi\left(A_{d_{1}-1}\right)$ - непересекающиеся подмножества в $I_{d_{1}-1}$, причем $\xi(A)=\xi\left(A_{0}\right) \cup \ldots \cup \xi\left(A_{d_{1}-1}\right)$. В частности, поскольку $\left|\xi\left(A_{j}\right)\right|=\left|A_{j}\right|$, $j \in \overline{0, d_{1}-1}$, получаем $|\xi(A)|=|A|$.

Лемма 3. Зафиксируем $n \in \xi\left(A_{j}\right)$ и пусть $m \in \xi(A)-$ maкое число, что $n \bmod$ $d_{i}^{a_{i}} \neq m \bmod d_{i}^{a_{i}}$ для всех $i \in \overline{2, t}$. Тогда $m \in \xi\left(A_{j}\right)$.

Доказательство. Пусть $n^{\prime} \in A_{j}$ - такое число, что $\xi\left(n^{\prime}\right)=n$ и $m^{\prime}$ - любое из таких чисел, что $\xi\left(m^{\prime}\right)=m$. Если $m \in \xi\left(A_{k}\right)$ при $k \neq j$, т.е. $m^{\prime} \in A_{k}$, то НОД $\left(n^{\prime}-m^{\prime}, d\right)=1$. Действительно, $d_{1}$ не делит разность $n^{\prime}-m^{\prime}$, поскольку $n^{\prime} \bmod d_{1}=j \neq k=m^{\prime} \bmod d_{1}$. Для любого $i \in \overline{2, t}$ число $d_{i}$ не делит разность $n^{\prime}-m^{\prime}$, поскольку

$$
\begin{aligned}
& n^{\prime} \bmod d_{i}=\left(n^{\prime} \bmod d_{i}^{a_{i}}\right) \bmod d_{i}= \\
& =\left(\left(n^{\prime}+\left(d_{1}-1\right)-\left(n^{\prime} \bmod d_{1}\right)-\left(d_{1}-1\right)+\left(n^{\prime} \bmod d_{1}\right)\right) \bmod d_{i}^{a_{i}}\right) \bmod d_{i}= \\
& =\left(\left(\xi\left(n^{\prime}\right)-\left(d_{1}-1\right)+j\right) \bmod d_{i}^{a_{i}}\right) \bmod d_{i}=\left(n-\left(d_{1}-1\right)+j\right) \bmod d_{i}
\end{aligned}
$$

и, аналогично, $m^{\prime} \bmod d_{i}=\left(m-\left(d_{1}-1\right)+k\right) \bmod d_{i}$. Отсюда получаем $(n-m) \bmod d_{i}=(k-j) \bmod d_{i}$, но $(k-j) \bmod d_{i} \neq 0$, т.к. $k, j<d_{1}<d_{i}$.

Значит, $n^{\prime}, m^{\prime} \in A$, но НОД $\left(n^{\prime}-m^{\prime}, d\right)=1$. Получаем противоречие с тем, что $A-$ множество нетривиальных разностей.

Пусть теперь множество $A$ не является элементарным и имеет мощность $w_{\max }(d)$. Тогда не менее двух множеств из $A_{0}, \ldots, A_{d_{1}-1}$ не пусты. Пусть это множества $A_{l}$ и $A_{\tilde{l}}, l \neq \tilde{l}$. Рассмотрим $n \in \xi\left(A_{l}\right)$ и $\tilde{n} \in \xi\left(A_{\tilde{l}}\right)$. Из леммы 2 следует, что $n \neq \tilde{n}$. Найдем число таких $m \in I_{d_{1}-1}$, что $m \bmod d_{i}^{a_{i}} \neq n \bmod d_{i}^{a_{i}}$ и $m \bmod d_{i}^{a_{i}} \neq \tilde{n} \bmod d_{i}^{a_{i}}$ для всех $i \in \overline{2, t}$. Для этого заметим: $m \bmod d_{1}=d_{1}-1$, т.е. $m \bmod d_{1}^{a_{1}}$ принимает $d_{1}^{a_{1}-1}$ возможных значений; при $i \in \overline{2, t} m \bmod d_{i}^{a_{i}}$ принимает любые значения от 0 до $d_{i}^{a_{1}}-1$, кроме $n \bmod d_{i}^{a_{i}}$ и $\tilde{n} \bmod d_{i}^{a_{i}}$, т.е. не менее $d_{i}^{a_{i}}-2$ значений. Поскольку по китайской теореме каждый набор остатков от деления на $d_{1}^{a_{1}}, \ldots, d_{t}^{a_{t}}$ однозначно задает число $m \in N_{d}$, таких $m$ существует $d_{1}^{a_{1}-1} \cdot \prod_{i=1}^{t-1}\left(d_{i}^{a_{i}}-2\right)$ штук. Теперь остается отметить, что любое из таких $m$ не лежит в $\xi(A)$, поскольку в противном случае, согласно лемме $3, m \in \xi\left(A_{l}\right)$ и $m \in \xi\left(A_{\tilde{l}}\right)$, что противоречит лемме 2. Учитывая, что $\xi(A) \subset I_{d_{1}-1}$, получаем

$$
\begin{gathered}
w_{\max }(d)=|A|=|\xi(A)| \leqslant\left|I_{d-1}-1\right|-d_{1}^{a_{1}-1} \cdot \prod_{i=2}^{t}\left(d_{i}^{a_{i}}-2\right) \\
=\frac{d}{d_{1}}-d_{1}^{a_{1}-1} \cdot \prod_{i=2}^{t}\left(d_{i}^{a_{i}}-2\right)=\frac{d}{d_{1}} \cdot\left(1-\prod_{i=2}^{t}\left(1-\frac{2}{d_{i}^{a_{i}}}\right)\right) .
\end{gathered}
$$

\section{3. Построение неэлементарных множеств нетривиальных разностей}

Заметим, что $I=\left\{n_{1}, \ldots, n_{r}\right\} \subset N_{d}$ с минимальным элементом $n_{1}$ является множеством нетривиальных разностей тогда и только тогда, когда множество 
$J=\left\{0, n_{2}-n_{1}, \ldots, n_{r}-n_{1}\right\}$ также является множеством нетривиальных разностей; более того, $I$ и $J$ одновременно являются или не являются элементарными. Из этого рассуждения, в частности, следует, что существует неэлементарное множество нетривиальных разностей мощности $w_{\max }(d)$, содержащее 0 . Поэтому, а также для простоты формулировок будем далее строить неэлементарные множества нетривиальных разностей, содержащие 0 .

Выберем в множестве $\left\{d_{1}, \ldots, d_{t}\right\}$ некоторый элемент $d_{i_{0}}$ и число $p \in \overline{1, d_{i_{0}}-1}$. Рассмотрим произвольное $A \subset \overline{1, t}, A \neq \varnothing, A \neq \overline{1, t}$, и свяжем с ним следующее множество $I_{A} \subset N_{d}$ : элемент $n \in N_{d}$ лежит в $I_{A}$ тогда и только тогда, когда $d_{i} \mid n$ при всех $i \in A, d_{i}$ не делит $n$, если $i \notin A$ и, если $i_{0} \notin A$, то $n \bmod d_{i_{0}}=p$. Получаем, что $n \in I_{A}$ тогда и только тогда, когда выполнены условия:

$$
n \bmod d_{i} \begin{cases}=0, & \text { если } i \in A, \\ \neq 0, & \text { если } i \in \overline{1, t} \backslash\left(A \cup\left\{i_{0}\right\}\right), \\ =p, & \text { если } i=i_{0} \notin A .\end{cases}
$$

Так как $A \neq \overline{1, t}$, то $0 \notin I_{A}$.

Для того чтобы найти мощность $I_{A}$, заметим: для $n \in I_{A}$ при $i \in A \cup\left\{i_{0}\right\}$ число $n \bmod d_{i}$ определено однозначно, поэтому величина $n \bmod d_{i}^{a_{i}}$ принимает $d_{i}^{a_{i}-1}$ значений; если $i \notin A \cup\left\{i_{0}\right\}$, то $n \bmod d_{i} \in\left\{1,2, \ldots, d_{i}-1\right\}$, поэтому $n \bmod d_{i}^{a_{i}}$ принимает $d_{i}^{a_{i}}-d_{i}^{a_{i}-1}=d_{i}^{a_{i}-1} \cdot\left(d_{i}-1\right)$ значений. Обозначим $\left|I_{A}\right|=r(A)$; по китайской теореме об остатках имеем

$$
\begin{aligned}
r(A) & =\left(\prod_{i \in A \cup\left\{i_{0}\right\}} d_{i}^{a_{i}-1}\right) \cdot\left(\prod_{i \in \overline{1, t} \backslash\left(A \cup\left\{i_{0}\right\}\right)}\left(d_{i}^{a_{i}-1} \cdot\left(d_{i}-1\right)\right)\right) \\
& =\frac{d}{d_{1} \cdot \ldots \cdot d_{t}} \cdot \prod_{i \in \overline{1, t} \backslash\left(A \cup\left\{i_{0}\right\}\right)}\left(d_{i}-1\right) .
\end{aligned}
$$

Из определения следует, что для разных непустых собственных подмножеств $A, B \subset \overline{1, t}$ множества $I_{A}$ и $I_{B}$ не пересекаются. Действительно, пусть $A \backslash B \neq \varnothing$ или $B \backslash A \neq \varnothing$; предположим первое, во втором случае действуем аналогично. Возьмем $i \in A \backslash B$, тогда $d_{i} \mid n$ для любого $n \in I_{A}$, но также $d_{i}$ не делит $m$ для любого $m \in I_{B}$. Значит, $I_{A} \cap I_{B}=\varnothing$.

Теперь рассмотрим некоторое семейство подмножеств $A_{1}, A_{2}, \ldots, A_{v} \subset \overline{1, t}$. Согласно сказанному выше, эта система задает подмножество $I\left(A_{1}, A_{2}, \ldots, A_{v}\right)=$ $\{0\} \cup \bigcup_{j=1}^{v} I_{A_{j}} \subset N_{d}$ мощности

$$
1+\sum_{j=1}^{v} r\left(A_{j}\right)=1+\frac{d}{d_{1} \cdot \ldots \cdot d_{t}} \cdot \sum_{j=1}^{v}\left(\prod_{i \in \overline{1, t} \backslash\left(A_{j} \cup\left\{i_{0}\right\}\right)}\left(d_{i}-1\right)\right) .
$$

При некоторых ограничениях на семейство $A_{1}, A_{2}, \ldots, A_{v}$ можно гарантировать, что соответствующее подмножество $N_{d}$ окажется неэлементарным множеством нетривиальных разностей. 
Утверждение 5. Пусть $A_{1}, A_{2}, \ldots, A_{v} \subset \overline{1, t}$ - такое семейство собственных непустых подмножеств, что для любых $l, j \in \overline{1, v}, l \neq j$, либо $A_{l} \cap A_{j} \neq \varnothing$, либо $i_{0} \notin A_{l} \cup A_{j}$. Тогда $I\left(A_{1}, A_{2}, \ldots, A_{v}\right)$ является множеством нетривиальных разностей. Кроме того, $I\left(A_{1}, A_{2}, \ldots, A_{v}\right)$ элементарно тогда и только тогда, когда $\bigcap_{j=1}^{v} A_{j} \neq \varnothing$.

Доказательство. В условиях утверждения рассмотрим $n, m \in I\left(A_{1}, A_{2}, \ldots, A_{v}\right)$. Если $n, m \in I_{A_{j}} \cup\{0\}$, то разность $n-m$ делится на все $d_{i}, i \in A_{j}$ (по условию $\left.A_{j} \neq \varnothing\right)$. Если $n \in I_{A_{j}}$ и $m \in I_{A_{l}}$, то возможны два случая. Если $A_{l} \cap A_{j} \neq \varnothing$, то существует $i \in A_{l} \cap A_{j}$, и тогда $d_{i} \mid n-m$. Если $i_{0} \notin A_{l} \cup A_{j}$, то $n \bmod d_{i_{0}}=$ $m \bmod d_{i_{0}}=p$ и поэтому $d_{i_{0}} \mid n-m$.

Пусть, далее, $I\left(A_{1}, A_{2}, \ldots, A_{v}\right)$ элементарно по модулю $d_{i}$, тогда $d_{i} \mid n-0$ для любого $n \in A_{j}, j \in \overline{1, v}$. Получаем, что $d_{i} \mid n$, тогда $i \in A_{j}, j \in \overline{1, v}$. Значит, $i \in \bigcap_{j=1}^{v} A_{j}$, т.е. $\bigcap_{j=1}^{v} A_{j} \neq \varnothing$. В обратную сторону: если $i \in \bigcap_{j=1}^{v} A_{j}$, то любое число из $I\left(A_{1}, A_{2}, \ldots, A_{v}\right)$ (включая, очевидно, 0$)$ делится на $d_{i}$ и, соответственно, любая разность делится на $d_{i}$.

Приведем пример семейства $A_{1}, A_{2}, \ldots, A_{v}$, удовлетворяющего условиям утверждения. Положим $i_{0}=1, p=1$. Рассмотрим семейство всех непустых подмножеств множества $\overline{2, t}$. Это семейство состоит из $v=2^{t-1}-1$ членов и удовлетворяет условиям утверждения 5 . Отметим, что в данном случае

$$
\begin{aligned}
& \sum_{j=1}^{v}\left(\prod_{i \in \overline{1, t} \backslash\left(A_{j} \cup\left\{i_{0}\right\}\right)}\left(d_{i}-1\right)\right)=\sum_{A \subset \overline{2, t}: A \neq \varnothing}\left(\prod_{i \in \overline{1, t} \backslash\left(A \cup\left\{i_{0}\right\}\right)}\left(d_{i}-1\right)\right) \\
& =\sum_{A \subset \overline{2, t: A \neq \overline{2, t}}}\left(\prod_{i \in A}\left(d_{i}-1\right)\right)=(*) .
\end{aligned}
$$

Каждое $A \subset \overline{2, t}$ однозначно соответствует своему характеристическому вектору $\left(\alpha_{2}, \ldots, \alpha_{t}\right) \in\{0,1\}^{t-1}: i \in A$ тогда и только тогда, когда $\alpha_{i}=1$. В этих терминах $(*)$ принимает вид

$$
\begin{gathered}
(*)=\sum_{\substack{\left(\alpha_{2}, \ldots, \alpha_{t}\right) \in\{0,1\}^{t-1} \\
\left(\alpha_{2}, \ldots, \alpha_{t}\right) \neq(1, \ldots, 1)}}\left(d_{2}-1\right)^{\alpha_{2}} \cdot \ldots \cdot\left(d_{t}-1\right)^{\alpha_{t}} . \\
\text { Заметив, что } \sum_{\left(\alpha_{2}, \ldots, \alpha_{t}\right) \in\{0,1\}^{t-1}} b_{2}^{\alpha_{2}} \cdot \ldots \cdot b_{t}^{\alpha_{t}}=\left(1+b_{2}\right) \cdot \ldots \cdot\left(1+b_{t}\right) \text {, получим: }
\end{gathered}
$$$$
(*)=d_{2} \cdot \ldots \cdot d_{t}-\left(d_{2}-1\right) \cdot\left(d_{3}-1\right) \cdot \ldots \cdot\left(d_{t}-1\right)=d_{2} \cdot \ldots \cdot d_{t} \cdot\left(1-\prod_{i=2}^{t}\left(1-\frac{1}{d_{i}}\right)\right),
$$

поэтому правая часть формулы (3) принимает вид $1+\frac{d}{d_{1}} \cdot\left(1-\prod_{i=2}^{t}\left(1-\frac{1}{d_{i}}\right)\right)$.

Для рассматриваемого семейства $A_{1}, A_{2}, \ldots, A_{v}$ выполнено $\bigcap_{j=1}^{v} A_{j}=\varnothing$. Поэтому $I\left(A_{1}, A_{2}, \ldots, A_{v}\right)$ является неэлементарным множеством нетривиальных разностей. Таким образом, в качестве следствия проведенных рассуждений получаем нижнюю оценку величины $w_{\max }(d)$. 
Следствие 1. Пусть число $d$ таково, что $t \geqslant 3$. Тогда

$$
w_{\max }(d) \geqslant 1+\frac{d}{d_{1}} \cdot\left(1-\prod_{i=2}^{t}\left(1-\frac{1}{d_{i}}\right)\right) .
$$

Это не единственный пример семейства $A_{1}, A_{2}, \ldots, A_{v}$, удовлетворяющего условиям утверждения 5. Например, положим $i_{0}=1, p=1$ и рассмотрим следующее семейство: $A_{1}=\{1, \ldots, r\}, A_{2}=\{1, r+1, r+2, \ldots, t\}, 2 \leqslant r \leqslant t-1, A_{3}, \ldots$, $A_{v}$ суть все такие подмножества в $\overline{2, t}$, что в каждом $A_{i}$ есть хотя бы одно число из $\overline{2, r}$ и хотя бы одно число из $\overline{r+1, t}$. Тогда условия утверждения 5 выполнены: $A_{1} \cap A_{2} \neq \varnothing$; если $j \in \overline{3, v}$, то $A_{1} \cap A_{j} \neq \varnothing$ и $A_{2} \cap A_{j} \neq \varnothing$; если $l, j \in \overline{3, v}, l \neq j$, то $1 \notin A_{l} \cup A_{j}$. Опуская громоздкие выкладки, которые аналогичны преобразованиям при выводе (4), приходим к равенству

$$
\begin{gathered}
\left|I\left(A_{1}, \ldots, A_{v}\right)\right|=1+\frac{d}{d_{1}}\left(1+\prod_{i=2}^{t}\left(1-\frac{1}{d_{i}}\right)\right. \\
\left.-\left(1-\frac{1}{d_{r+1} \cdot \ldots \cdot d_{t}}\right) \cdot \prod_{i=2}^{r}\left(1-\frac{1}{d_{i}}\right)-\left(1-\frac{1}{d_{2} \cdot \ldots \cdot d_{r}}\right) \cdot \prod_{i=r+1}^{t}\left(1-\frac{1}{d_{i}}\right)\right) .
\end{gathered}
$$

Несложно проверить, что полученное значение меньше оценки (4). Можно предложить следующую гипотезу: неравенство (4) на самом деле является равенством.

\section{4. Качественное сравнение верхней и нижней оценок}

В предыдущих разделах доказаны следующие оценки при $t \geqslant 3$ :

$$
1-\prod_{i=2}^{t}\left(1-\frac{2}{d_{i}^{a_{i}}}\right) \geqslant \frac{w_{\max }(d)}{d / d_{1}}>1-\prod_{i=2}^{t}\left(1-\frac{1}{d_{i}}\right)
$$

Согласно утверждению 1 , мощность наибольшего множества нетривиальных разностей равна $d / d_{1}$. Поэтому оценки (5) можно трактовать как оценки того, насколько мощность неэлементарного множества может быть близка к мощности наибольшего множества нетривиальных разностей. Чтобы оценить качество оценок (5), потребуется оценить снизу произведение $\prod_{i=2}^{t}\left(1-2 / d_{i}^{a_{i}}\right)$ и сверху произведение $\prod_{i=2}^{t}\left(1-1 / d_{i}\right)$ функциями более простого вида. В принципе, асимптотическое поведение указанных произведений при $t \rightarrow \infty$ известно, см., например, [1]. В этом параграфе уделим внимание оценкам, выполняющимся при всех $t \geqslant 3$; будем далее предполагать это условие выполненным.

Нижняя оценка в (5) от $a_{i}$ не зависит. Избавимся от $a_{i}$ и в верхней оценке: $\prod_{i=2}^{t}\left(1-2 / d_{i}^{a_{i}}\right) \geqslant \prod_{i=2}^{t}\left(1-2 / d_{i}\right)$. Отсюда получаем

$$
1-\prod_{i=2}^{t}\left(1-\frac{2}{d_{i}}\right) \geqslant \frac{w_{\max }(d)}{d / d_{1}}>1-\prod_{i=2}^{t}\left(1-\frac{1}{d_{i}}\right)
$$


Далее рассмотрим три случая: в первом не накладываем никаких ограничений на значения $d_{2}, \ldots, d_{t}$; во втором рассмотрим значения $d_{i}=p_{i}, i=\overline{1, t}$, где $p_{i}-i$-е по счету простое число; в третьем случае предположим, что $d_{i}$ растут пропорционально $p_{i}$, т.е. существуют такие константы $a>b>1$, что $b \cdot p_{i} \leqslant d_{i} \leqslant a \cdot p_{i}$ для любого $i \in \overline{2, t}$.

I. Значения $2<d_{2}<\ldots<d_{t}-$ произвольные простые числа.

Для этого случая получим оценки величины $\frac{w_{\max }(d)}{d / d_{1}}$ функциями от $\sum_{i=2}^{t} 1 / d_{i}$ (следствие 2) и функциями от $d_{2}, d_{t}$ (утверждение 6).

Лемма 4. Пусть $r_{0}, r_{1}, r_{2}, \ldots, r_{n}$ - положительные числа, причем $r_{i} \geqslant r_{0}>1$, $i=\overline{1, n}$. Тогда верны оценки $e^{-C_{0} \cdot S} \leqslant \prod_{i=1}^{n}\left(1-1 / r_{i}\right) \leqslant e^{-S}$, где $S=\sum_{i=1}^{n} 1 / r_{i}, C_{0}=$ $\frac{2 r_{0}-1}{2\left(r_{0}-1\right)}$.

Доказательство. Пусть $P=\prod_{i=1}^{n}\left(1-1 / r_{i}\right)$, тогда $P=e^{-S_{0}}$, где $S_{0}=$ $\sum_{i=1}^{n} \ln \left(1-1 / r_{i}\right)$. Так как $-\ln (1-t)=t+t^{2} / 2+t^{3} / 3+\ldots$ при $1>t \geqslant 0$, то $t \leqslant-\ln (1-t) \leqslant t+t^{2} / 2+t^{3} / 2+\ldots=t+\frac{t^{2} / 2}{1-t}$. При $t=1 / r_{i} \leqslant 1 / r_{0}$ имеем

$$
t+\frac{t^{2} / 2}{1-t} \leqslant t+\frac{1 / r_{0}}{2\left(1-1 / r_{0}\right)} \cdot t=t \cdot \frac{2 r_{0}-1}{2\left(r_{0}-1\right)}=C_{0} \cdot t .
$$

Следовательно, $-C_{0} \cdot t \leqslant \ln (1-t) \leqslant-t$. Применяя это неравенство к сумме $S_{0}$, доказываем лемму.

Сначала положим $r_{i}=d_{i+1}, i=\overline{1, t-1}$; тогда $\prod_{i=2}^{t}\left(1-1 / d_{i}\right) \leqslant e^{-S_{1}}$, где $S_{1}=$ $\sum_{i=1}^{t-1} 1 / d_{i+1}$. Теперь положим $r_{i}=d_{i+1} / 2, i=\overline{1, t-1}, r_{0}=d_{2} / 2$; тогда $C_{0}=\frac{d_{2}-1}{d_{2}-2}$ и $\prod_{i=2}^{t}\left(1-2 / d_{i}\right) \geqslant e^{-C_{0} \cdot S_{2}}$, где $S_{2}=\sum_{i=1}^{t-1} 2 / d_{i+1}=2 \cdot S_{1}$. Таким образом, доказано следующее утверждение.

Следствие 2. Верны оценки

$$
1-e^{-\left(2 \cdot \frac{d_{2}-1}{d_{2}-2} \cdot\right) S_{1}} \geqslant \frac{w_{\max }(d)}{d / d_{1}}>1-e^{-S_{1}},
$$

где $S_{1}=1 / d_{2}+1 / d_{3}+\ldots+1 / d_{t}$.

Из условия $d_{2} \geqslant 3$ следует, что $\frac{d_{2}-1}{d_{2}-2} \leqslant 2$, поэтому, в частности, левое неравенство можно заменить более грубым, но более простым неравенством $1-e^{-4 S_{1}} \geqslant \frac{w_{\max }(d)}{d / d_{1}}$.

Теперь рассмотрим возможность получения простых оценок, зависящих только от $d_{t}$ (верхняя) или только от $d_{2}$ (нижняя). В случае I можно предложить только элементарные оценки, причем неулучшаемые.

Утверждение 6. Верны оценки

$$
\left(1-2 / d_{2}\right)^{t-1} \leqslant \prod_{i=2}^{t}\left(1-2 / d_{i}\right)<\prod_{i=2}^{t}\left(1-1 / d_{i}\right) \leqslant\left(1-1 / d_{t}\right)^{t-1}
$$


Эти оценки не улучшаемы в том смысле, что для любых $t \geqslant 3, \alpha>1$ и $0<\beta<1$ найдутся такие простые числа $d_{2}<\ldots<d_{t}$, что $\prod_{i=2}^{t}\left(1-2 / d_{i}\right)<\alpha \cdot\left(1-2 / d_{2}\right)^{t-1}$ $u \prod_{i=2}^{t}\left(1-1 / d_{i}\right)>\beta \cdot\left(1-1 / d_{t}\right)^{t-1}$.

Доказательство. Оценки (7) очевидны.

Рассмотрим произвольные $t \geqslant 3, \alpha>1$ и $0<\beta<1$. Возьмем функцию $\pi(x)$, равную количеству простых чисел, не превышающих $x$. Известна оценка ([2]) $\pi(2 x)-\pi(x)>0,6 x / \ln x$, верная при всех $x \geqslant 21$. Возьмем такое большое значение $d_{t} \geqslant 43$, что $\pi\left(d_{t}\right)-\pi\left(d_{t} / 2+1\right)>t-1$. Поскольку функция $0,6 x / \ln x$ неограниченно возрастает, такое $d_{t}$ найдется. Значит, среди чисел $d_{t} / 2+1, d_{t} / 2+2, \ldots, d_{t}-1$ найдется не менее $t-2$ различных простых чисел. Пусть это числа $d_{2}<\ldots<d_{t-1}$, тогда $d_{i}>d_{t} / 2, i=\overline{2, t}$. Из последнего неравенства следует, что $\prod_{i=2}^{t}\left(1-1 / d_{i}\right)>$ $\left(1-2 / d_{t}\right)^{t-1}$. Так как $\frac{1-2 / d_{t}}{1-1 / d_{t}}=1-\frac{1}{d_{t}-1}$, то $\left(1-\frac{2}{d_{t}}\right)^{t-1}=\left(1-\frac{1}{d_{t}}\right)^{t-1} \cdot\left(1-\frac{1}{d_{t}-1}\right)^{t-1}$. Положим $B_{t}=1-\frac{1}{d_{t}-1}$, тогда $\prod_{i=2}^{t}\left(1-1 / d_{i}\right)>\left(1-1 / d_{t}\right)^{t-1} \cdot B_{t}^{t-1}$.

Из способа выбора $d_{2}, \ldots, d_{t}$ следует, что $2 d_{2}>d_{t}$, поэтому $d_{2}<d_{3}<\ldots<$ $d_{t}<2 d_{2}$. Тогда $\prod_{i=2}^{t}\left(1-2 / d_{i}\right)<\left(1-1 / d_{2}\right)^{t-1}$. Далее, $\frac{1-1 / d_{2}}{1-2 / d_{2}}=1+\frac{1}{d_{2}-2}$. Положим $A_{t}=1+\frac{1}{d_{2}-2}$, тогда $\prod_{i=2}^{t}\left(1-2 / d_{i}\right)<\left(1-2 / d_{2}\right)^{t-1} \cdot A_{t}^{t-1}$.

При увеличении $d_{t}$ в силу неравенства $2 d_{2}>d_{t}$ растет также и $d_{2}$. Увеличим $d_{t}$ еще больше, чтобы выполнялись условия $B_{t}>\beta^{1 /(t-1)}$ и $A_{t}<\alpha^{1 /(t-1)}$. Тогда окажутся выполненными требуемые неравенства.

II. Eсли $i=\overline{2, t}$, mо $d_{i}=p_{i}$, где $p_{i}-i$-е по счету простое число.

Поскольку $d_{1}<d_{2}=3$, в данном случае $d_{1}=2$. Получим оценки в виде функций от $p_{t}$ (утверждение 7 ), а затем в виде функций от $t$ (следствие 3 ).

Утверждение 7. Верны оценки

$$
1-\frac{0,518}{\ln ^{2} p_{t}} \geqslant \frac{w_{\max }(d)}{d / 2}>1-\frac{1,125}{\ln p_{t}} .
$$

Доказательство. Сначала докажем верхнюю оценку. Из (6) и условий п. II следует, что $\prod_{i=2}^{t}\left(1-2 / p_{i}\right) \leqslant 1-\frac{w_{\max }(d)}{d / 2}$. Имеем $\prod_{i=2}^{t}\left(1-2 / p_{i}\right)=e^{Q_{1}(t)+Q_{2}(t)}$, где $Q_{1}(t)=\sum_{i=2}^{t}\left(-2 / p_{i}\right), Q_{2}(t)=\sum_{i=2}^{t}\left(\ln \left(1-2 / p_{i}\right)+2 / p_{i}\right)$. Оценим снизу каждое из $Q_{1}(t), Q_{2}(t)$. Для первой функции, используем известную оценку из [2]: $\sum_{i=1}^{t} 1 / p_{i}<$ $\ln \ln p_{t}+0,261498+1 / \ln ^{2} p_{t}$, находим, что

$$
Q_{1}(t)=-\sum_{i=2}^{t} \frac{2}{p_{i}}=(-2) \cdot\left(-\frac{1}{2}+\sum_{i=1}^{t} \frac{1}{p_{i}}\right)>0,477004-2 \cdot\left(\ln \ln p_{t}+\frac{1}{\ln ^{2} p_{t}}\right) .
$$


Теперь оценим снизу $Q_{2}(t)$. Имеет место неравенство $\ln (1+s) \leqslant s$, верное при $s>-1$, причем неравенство обращается в равенство только при $s=0$. Так как $i \geqslant 2$, то $p_{i} \geqslant 3$, поэтому $-2 / p_{i}>-1$. Отсюда $\ln \left(1-2 / p_{i}\right)+2 / p_{i}<0$. Значит, $Q_{2}(t)>\sum_{i=2}^{\infty}\left(\ln \left(1-2 / p_{i}\right)+2 / p_{i}\right)$. Разделим последнюю сумму на две: $Q_{2,1}=\sum_{i=2}^{10^{6}}\left(\ln \left(1-2 / p_{i}\right)+2 / p_{i}\right)$ и $Q_{2,2}=\sum_{i=10^{6}+1}^{\infty}\left(\ln \left(1-2 / p_{i}\right)+2 / p_{i}\right)$. Первая сумма оценена при помощи ЭВМ: $e^{Q_{2,1}} \leqslant 0,516638$. Далее воспользуемся полученным в лемме 4 неравенством $\ln (1-t) \geqslant-\left(t+\frac{t^{2} / 2}{1-t}\right)$. Согласно этому неравенству $\ln \left(1-2 / p_{i}\right)+2 / p_{i} \geqslant-\frac{2 / p_{i}^{2}}{1-2 / p_{i}}$ при всех $i>10^{6}$. Из условия $p_{i}>3$ следует, что $1-2 / p_{i}>1 / 3$. Отсюда $-\frac{2 / p_{i}^{2}}{1-2 / p_{i}} \geqslant-6 / p_{i}^{2}>-6 / i^{2}$. Следовательно, $Q_{2,2} \geqslant-6 \cdot \sum_{i=2}^{t} 1 / i^{2}$. Воспользуемся неравенством $\frac{1}{i^{2}} \leqslant \int_{i-1}^{i} \frac{d r}{r^{2}}$, тогда $-\sum_{i=10^{6}+1}^{\infty} 1 / i^{2} \geqslant$ $-\sum_{i=10^{6}+1}^{\infty} \int_{i-1}^{i} \frac{d r}{r^{2}}=-\int_{10^{6}}^{\infty} \frac{d r}{r^{2}}=\frac{1}{10^{6}}$. Значит, $Q_{2,2}(t) \geqslant-6 \cdot 10^{-6}$. Таким образом, получаем $e^{Q_{2}(t)}>0,516638 \cdot 0,999994>0,516634$.

Из полученного неравенства и оценки (9) следует, что

$$
\prod_{i=2}^{t}\left(1-2 / p_{i}\right)=e^{Q_{1}(t)+Q_{2}(t)} \geqslant 0,832421 \cdot \frac{1}{\ln ^{2} p_{t}} \cdot e^{-2 / \ln ^{2} p_{t}} .
$$

Воспользуемся неравенством $e^{y} \geqslant 1+y$, верным при всех вещественных $y$. Получим оценку

$$
\prod_{i=2}^{t}\left(1-2 / p_{i}\right) \geqslant 0,832421 \cdot \frac{1}{\ln ^{2} p_{t}} \cdot\left(1-\frac{2}{\ln ^{2} p_{t}}\right) .
$$

Докажем неравенство

$$
\prod_{i=2}^{t}\left(1-2 / p_{i}\right) \geqslant \frac{0,518}{\ln ^{2} p_{t}}
$$

из которого будет следовать доказываемая верхняя оценка величины $\frac{w_{\max }(d)}{d / 2}$. Это неравенство проверяется непосредственным вычислением на ЭВМ при $t=3,4$. При $t \geqslant 5$ имеем $p_{t} \geqslant 11$ и поэтому

$$
0,832421 \cdot\left(1-\frac{2}{\ln ^{2} p_{t}}\right)>0,832421 \cdot\left(1-\frac{2}{\ln ^{2}(11)}\right) \approx 0,542 \ldots>0,518 .
$$

Из последнего неравенства и (10) получаем требуемое.

Перейдем к доказательству верхней оценки. Согласно (6) и условию II выполнено $1-\frac{w_{\max }(d)}{d / 2}<\prod_{i=2}^{t}\left(1-1 / p_{i}\right)$. В [2] приведена оценка $\prod_{p \leqslant x}\left(1-\frac{1}{p}\right)<\frac{\lambda}{\ln x}\left(1+\frac{1}{2 \ln ^{2} x}\right)$, где 
$\lambda=0,56145 \ldots$ Отсюда

$$
\begin{aligned}
& \prod_{i=2}^{t}\left(1-1 / p_{i}\right)=\prod_{2<p \leqslant p_{t}}\left(1-1 / p_{i}\right)=2 \prod_{2 \leqslant p \leqslant p_{t}}\left(1-1 / p_{i}\right)< \\
& <\frac{2 \cdot \lambda}{\ln p_{t}} \cdot\left(1+\frac{1}{2 \ln ^{2} p_{t}}\right)<\frac{1,12292}{\ln p_{t}} \cdot\left(1+\frac{1}{2 \ln ^{2} p_{t}}\right) .
\end{aligned}
$$

Докажем неравенство

$$
\prod_{i=2}^{t}\left(1-1 / p_{i}\right)<\frac{1,125}{\ln p_{t}}
$$

из которого будет следовать доказываемая верхняя оценка. Это неравенство проверяется непосредственным вычислением на ЭВМ при $p_{t}<2 \cdot 10^{7}$. При $p_{t}>2 \cdot 10^{7}$ получаем

$$
1,12292 \cdot\left(1+\frac{1}{2 \ln ^{2} p_{t}}\right)<1,12292 \cdot\left(1+\frac{1}{2 \ln ^{2}\left(2 \cdot 10^{7}\right)}\right) \approx 1,12490 \ldots<1,125
$$

Значит, $\frac{1,125}{\ln p_{t}}>\frac{1,12292}{\ln p_{t}} \cdot\left(1+\frac{1}{2 \ln ^{2} p_{t}}\right)$ при $p_{t}>2 \cdot 10^{7}$, далее из (12) следует доказываемое неравенство.

Следствие 3. Верны оценки

$$
1-\frac{0,284}{\ln ^{2}(t \ln t)} \geqslant \frac{w_{\max }(d)}{d / 2}>1-\frac{1,125}{\ln (t \ln t)} .
$$

Доказательство. Воспользуемся известными оценками значения $p_{t}$ в терминах функций от $t$, приведенных в [2]. При всех $t \geqslant 2$ верна нижняя оценка $p_{t}>t \ln t$. Доказываемая нижняя оценка получается при подстановке этого неравенства в правую часть (8).

При $3 \leqslant t \leqslant 8$ непосредственной проверкой убеждаемся в истинности неравенства $1-\frac{0,284}{\ln ^{2}(t \ln t)}>1-\prod_{i=2}^{t}\left(1-2 / p_{i}\right)$. Из левой части неравенств (6) для указанных значений $t$ следует требуемое неравенство $1-\frac{0,284}{\ln ^{2}(t \ln t)} \geqslant \frac{w_{\max }(d)}{d / 2}$. Пусть далее $t \geqslant 9$. Известно [2], что при таких $t$ верна верхняя оценка $p_{t}<t(\ln t+\ln \ln t)$. Подставив эту оценку в левую часть неравенства (8), получим

$$
\frac{w_{\max }(d)}{d / 2}<1-\frac{0,518}{\ln ^{2}(t(\ln t+\ln \ln t))} .
$$

Докажем, что $\ln ^{2}(t(\ln t+\ln \ln t))<1,823 \cdot \ln ^{2}(t \ln t)$. Тогда требуемая верхняя оценка будет доказана ввиду неравенств

$$
1-\frac{0,518}{\ln ^{2}(t(\ln t+\ln \ln t))}<1-\frac{0,518}{1,823 \cdot \ln ^{2}(t \ln t)}<1-\frac{0,284}{\ln ^{2}(t \ln t)},
$$

поскольку $0,518 / 1,823=0,28414 \ldots$ 
Обозначим $W(t)=\ln ^{2}(t(\ln t+\ln \ln t))$ и, используя неравенство $\ln (1+x)<x$, верное при $x>0$, получаем

$$
\begin{aligned}
& W(t)=\ln ^{2}\left(t \ln t\left(1+\frac{\ln \ln t}{\ln t}\right)\right)=\left(\ln (t \ln t)+\ln \left(1+\frac{\ln \ln t}{\ln t}\right)\right)^{2}< \\
& <\left(\ln (t \ln t)+\frac{\ln \ln t}{\ln t}\right)^{2} .
\end{aligned}
$$

Покажем, что $\frac{\ln \ln t}{\ln t}<0,35 \ln (t \ln t)$, тогда

$$
\left(\ln (t \ln t)+\frac{\ln \ln t}{\ln t}\right)^{2}<(1,35 \cdot \ln (t \ln t))^{2}<1,823 \cdot \ln ^{2}(t \ln t),
$$

что достаточно для доказательства следствия.

С помощью обозначения $x=\ln t \geqslant \ln 9$ требуемое неравенство запишется в виде $\frac{\ln x}{x}<0,35(x+\ln x)$ или $\ln x<0,35\left(x^{2}+x \ln x\right)$. Поскольку $\ln x<x$ при $x>0$, то достаточно доказать $x<0,35\left(x^{2}+x \ln x\right)$. После преобразования получаем $1-0,35 x<0,35 \ln x$. Но

$$
1-0,35 x<1-0,35 \ln 9 \approx 0,2309 \ldots<0,2755 \approx 0,35 \ln \ln 9<0,35 \ln x .
$$

III. Существуют такие $a>b>1$, что $b \cdot p_{i} \leqslant d_{i} \leqslant a \cdot p_{i}$ для любого $i \in \overline{2, t}$. В условиях этого случая неравенства (6) принимают вид

$$
1-\prod_{i=2}^{t}\left(1-\frac{2}{b p_{i}}\right) \geqslant \frac{w_{\max }(d)}{d / d_{1}}>1-\prod_{i=2}^{t}\left(1-\frac{1}{a p_{i}}\right)
$$

Утверждение 8. Верны неравенства

$$
1-\left(\frac{0,863}{\ln p_{t}}\right)^{4 / b}>\frac{w_{\max }(d)}{d / d_{1}}>1-\left(\frac{\sqrt{e}}{\ln p_{t}}\right)^{1 / a} .
$$

Доказательство. Воспользуемся леммой 4 для значений $r_{i}=a p_{i+1}, r_{0}=3 a \leqslant r_{i}=$ $a p_{i+1}, i=\overline{1, t-1}$. Кроме того, используем неравенство $\sum_{i=1}^{t} 1 / p_{i}>\ln \ln p_{t}$ из [2]. Имеем

$$
\prod_{i=2}^{t}\left(1-\frac{1}{a p_{i}}\right) \leqslant e^{-\sum_{i=2}^{t} \frac{1}{a p_{i}}}=\left(e^{-1 / 2+\sum_{i=1}^{t} 1 / p_{i}}\right)^{-1 / a}<\left(e^{-1 / 2+\ln \ln p_{t}}\right)^{-1 / a}=\left(\frac{\sqrt{e}}{\ln p_{t}}\right)^{1 / a} .
$$

Из правой части неравенств (14) получаем доказываемую нижнюю оценку.

Аналогично воспользуемся леммой 4 для значений $r_{i}=b\left(p_{i+1} / 2\right), r_{0}=1,5 b \leqslant$ $r_{i}=b\left(p_{i+1} / 2\right), i=\overline{1, t-1}$. Тогда $C_{0}=\frac{3 b-1}{3 b-2}$ и

$$
\prod_{i=2}^{t}\left(1-\frac{2}{b p_{i}}\right) \geqslant e^{-C_{0} \sum_{i=2}^{t} 2 / b p_{i}}=\left(e^{-1 / 2+\sum_{i=1}^{t} 1 / p_{i}}\right)^{-\frac{2 C_{0}}{b}} .
$$


Для суммы $\sum_{i=1}^{t} 1 / p_{i}$ известна [2] верхняя оценка $\sum_{i=1}^{t} 1 / p_{i}<\ln \ln p_{t}+0,2615+\frac{1}{\ln ^{2} p_{t}}$. Так как $t \geqslant 3$, то $p_{t} \geqslant 5$, поэтому $\sum_{i=1}^{t} 1 / p_{i}<\ln \ln p_{t}+0,2615+\frac{1}{\ln ^{2} 5}<\ln \ln p_{t}+0,648$. Получаем

$$
e^{-1 / 2+\sum_{i=1}^{t} 1 / p_{i}}>e^{-1 / 2+\ln \ln p_{t}+0,648}=e^{\ln \ln p_{t}+0,148}>1,159 \cdot \ln p_{t}>\frac{\ln p_{t}}{0,863} .
$$

Остается заметить, что $\frac{2}{b} \cdot C_{0}=\frac{2}{b} \cdot \frac{3 b-1}{3 b-2}<\frac{4}{b}$, поскольку последнее неравенство эквивалентно $b>1$. Применяя полученные неравенства к левой части (14), доказываем утверждение.

\section{Список литературы}

1. Prachar K., Primzahlverteilung, Springer-Verlag, Berlin Göttingen Heidelberg, 1957; пер. с нем.: Прахар К., Распределение простых чисел, М.: Мир, 1967, 513 с.

2. Rosser J., Schoenfeld L., "Approximate formulas for some functions of prime numbers", Ill. J. Math, 6:1 (1962), 64-94..

3. Stinson D.R. Muir J.A., "On the low Hamming weight discrete logarithm problem for nonadjacent representations.", Appl. Alg. in Eng., Commun. and Comput., 16:6 (2006), 461-472..

Статья поступила 17.02.2016. 